\title{
Rektumkarzinomchirurgie in Deutschland - eine 10-Jahres-Übersicht auf der Grundlage der Daten des AN-Instituts für Qualitätssicherung in der operativen Medizin gGmbH an der Otto-von-Guericke-Universität Magdeburg*
}

\author{
Rectal Cancer Surgery in Germany - a 10-Year-Analysis Based on the Data of the "Institute of \\ Quality Assurance in Operative Medicine" at the Otto-von-Guericke University Magdeburg
}

Autoren

Institute
H. Ptok ${ }^{1}$, A. Mundt ${ }^{2}$, H. Lippert ${ }^{1,3}$, I. Gastinger ${ }^{1}$

${ }^{1}$ AN-Institut für Qualitätssicherung in der operativen Medizin an der O-v-G-Universität Magdeburg, Magdeburg, Deutschland

${ }^{2}$ Klinik für Anaesthesie und Intensivmedizin, Carl-Thiem-Klinikum Cottbus, Cottbus, Deutschland

${ }^{3}$ Klinik für Allgemein-, Gefäß- und Viszeralchirurgie, Universitätsklinikum Magdeburg, Magdeburg, Deutschland

\section{Schlüsselwörter \\ - Rektumkarzinom \\ - multimodale Behandlung \\ - totale mesorektale Exzision \\ - Lokalrezidivrate \\ - Gesamtüberleben \\ - Beobachtungsstudie \\ Key words \\ - rectal cancer \\ - multimodal treatment \\ - total mesorectal excision \\ - local recurrence rate \\ - overall survival \\ - observational study}

\section{Bibliografie}

Dol http://dx.doi.org/

10.1055/s-0032-1328353

Online-publiziert 03.06.2013

Zentralbl Chir 2013; 138:

418-426 @ Georg Thieme

Verlag KG Stuttgart . New York .

ISSN 0044-409X

\section{Korrespondenzadresse}

Dr. Henry Ptok

AN-Institut für Qualitäts-

sicherung in der operativen

Medizin an der O-v-G-

Universität Magdeburg

Leipziger Straße 44

39120 Magdeburg

Deutschland

Tel.: 0391/6714068

henryptok@aol.com

\section{Zusammenfassung}

\section{V}

Hintergrund: Die Behandlung des Rektumkarzinoms hat sich in den letzten 2 Dekaden deutlich gewandelt. Durch konsequenten Einsatz neoadjuvanter Therapieverfahren und Anwendung der totalen mesorektalen Exzision beim tief sitzenden Rektumkarzinom konnte die lokale Tumorkontrolle signifikant gebessert werden. Mit der vorliegenden Analyse sollen die Umsetzung der multimodalen Therapie beim Rektumkarzinom unter den Bedingungen der Routineversorgung sowie die erreichten Ergebnisse über einen 10-JahresZeitraum untersucht werden.

Methode: Es wurden die Daten der prospektiven multizentrischen Beobachtungsstudie "Qualitätssicherung - Rektumkarzinom“ der Jahre 2000 bis 2010 ausgewertet. $\mathrm{N}=33724$ Patienten wurden erfasst. Die Resektionsrate betrug 95,2\%. Die Rate kurativer Resektionen betrug $84,2 \%$.

Ergebnisse: Die postoperative Gesamtmorbidität und Letalität zeigten keine Änderung im Verlauf der Beobachtung. Der Anteil neoadjuvant behandelter Patienten mit kurativer Resektion stieg von 5,6\% (2000) auf 40,5\% (2010). Die TME-Rate stieg bei tiefsitzenden Karzinomen von 75,2\% (2000) auf 95,3\% (2010). Für Patienten, die in den Jahren 2000/2001 kurativ reseziert wurden, lag die 5-Jahres-Lokalrezidivrate bei $11,7 \%$, während diese für in den Jahren 2005/2006 resezierte Patienten bei $4,6 \% \operatorname{lag}(p<0,001)$. Eine Verbesserung des Gesamtüberlebens zeigte sich nicht.

Schlussfolgerung: Bei gleichbleibender Gesamtmorbidität und Letalität hat der zunehmende Einsatz neoadjuvanter Behandlungen und die Etablierung der TME in der flächendeckenden Routineversorgung von Patienten mit Rektumkarzinomen zu einer signifikanten Verbesserung der lokalen Tumorkontrolle geführt, ohne dass ein Einfluss auf das Gesamtüberleben der Patienten nachweisbar ist.

\section{Abstract \\ $\nabla$}

Background: The treatment of rectal cancer has undergone pronounced changes during the last two decades. There has been a significant improvement in local tumour control due to consequent use of neo-adjuvant therapy and total mesorectal excision in cases of distal rectal cancer. The presented analysis examines the realisation of the multimodal therapy for rectal cancer under the conditions of routine patient-centred care over a period of ten years.

Method: The data acquired in the prospective multicentre observational study "Quality Assurance - Rectal Cancer" from the years 2000 to 2010 were analysed. $\mathrm{N}=33,724$ patients were documented. The resection rate was $95.2 \%$. The rate of curative resection was $84.2 \%$.

Results: No change was detected in perioperative total morbidity and lethality during the course of the study. The percentage of patients with neoadjuvant treatment and curative resection rose from $5.6 \%$ (2000) to $40.5 \%$ (2012). The rate of performed TME in distal rectal cancer rose from $75.2 \%$ (2000) to $95.3 \%$ (2012). For patients who underwent curative resection in the years 2000/ 2001 the 5-year local recurrence rate was $11.7 \%$, while it was found to be $4.6 \%$ for patients who were thus treated in the years 2005/2006 $(p<0.001)$. There was no improvement of total survival.

Conclusion: While an increase in the use of neoadjuvant treatment for rectal cancer and the establishment of TME in routine patient-centred care have led to a significant improvement in local tumour control with a constant total morbidity and lethality, there is no detectable influence on the patients' total survival. 


\section{Einleitung}

In den letzten Jahrzehnten hat sich die Behandlung des Rektumkarzinoms grundlegend gewandelt. Wesentlich beeinflusst durch die Ende der 1980er-Jahre von Heald publizierten hervorragenden Ergebnisse zur Verbesserung der lokalen Tumorkontrolle des Rektumkarzinoms durch die totale mesorektale Exzision (TME) [1] sowie die im Rahmen prospektiver randomisierter Studien ermittelten Ergebnisse zur Verbesserung der lokalen Tumorkontrolle durch eine neoadjuvante Bestrahlung oder Radiochemotherapie beim Rektumkarzinom [2-5] mit resultierenden multimodalen Behandlungskonzepten, konnten in den letzten Jahrzehnten die Ergebnisse der lokalen Behandlung des Rektumkarzinoms entscheidend verbessert werden. So berichteten Autoren noch Ende der '80er-Jahre über 5-Jahres-Lokalrezidivraten um $20 \%$ bis $25 \%$ [6-8], während heute die Lokalrezidivraten nach 5 und selbst nach 10 Jahren unter $10 \%$ angegeben werden [5,9]. Die lokale Tumorresektion nach dem Prinzip der TME mit einem negativen zirkumferenziellen Resektionsrand ( $\mathrm{CRM}^{-}$-Resektion) mit guter Qualität der TME [10-12] stellen heute die onkologisch entscheidenden Surrogatparameter dar und erlauben eine unmittelbar postchirurgische Bewertung der Qualität der lokalen Tumorbehandlung nach primärer Chirurgie oder multimodaler Therapie bei fortgeschrittenen Befunden.

Neben den onkologischen Ergebnissen stellen die postoperative Morbidität und Letalität wesentliche Qualitätsparameter dar. In funktioneller Hinsicht sind weiterhin die Rate an Harnblasenentleerungsstörungen sowie - nach kontinenzerhaltender Resektion - die Rate an postoperativen Anastomoseninsuffizienzen relevant [13-16].

Im Rahmen einer prospektiven multizentrischen Beobachtungsstudie zur Evaluation der flächendeckenden Versorgung von Patienten mit einem Rektumkarzinom in Deutschland wurden die früh-postoperativen und onkologischen Langzeitergebnisse der Rektumkarzinombehandlung seit 2000 untersucht. In der vorliegenden Arbeit werden die Entwicklungen hinsichtlich Patientendemografie, Tumordiagnostik und -therapie sowie die resultie- renden Ergebnisse über einen 10-Jahres-Verlauf in der flächendeckenden Versorgung dargestellt und diskutiert.

\section{Methode \\ $\nabla$}

Seit dem 01.01.2000 werden im Rahmen der prospektiven multizentrischen Beobachtungsstudien „Qualitätssicherung Kolon/ Rektum Karzinom (Primärtumor)“ (2000-2004) und „Qualitätssicherung Rektumkarzinom (Primärtumor - Elektivoperation) (seit 2005), organisiert und durchgeführt am AN-Institut für Qualitätssicherung in der operativen Medizin an der Otto-vonGuericke-Universität in Magdeburg, Daten von Patienten mit Rektumkarzinom erfasst. Der Dokumentationsbogen enthält 68 Items zur Erfassung demografischer Daten, Daten zur prätherapeutischen Diagnostik, behandlungsassoziierter Daten sowie Daten zur Tumorhistologie und zu den früh-postoperativen Ergebnissen. Weiterhin wurden regelmäßig (jährlich) Daten zum onkologischen Langzeitverlauf (Follow-up) erhoben. Die Teilnahme an der multizentrischen Studie war für die teilnehmenden Kliniken freiwillig. Es beteiligten sich Kliniken aller Versorgungsstufen. Eine ausführliche Beschreibung der Methode der Datenerhebung und Auswertung ist bereits publiziert [17].

In der vorliegenden Arbeit wurden ausgewählte patientenassoziierte (Alter, ASA-Score), tumorassoziierte (pT-Kategorie, UICCStadien), behandlungsassoziierte (Resektionsrate, kurative Resektionsrate, neoadjuvante Therapie, TME-Rate) Parameter, sowie ausgewählte Ergebnisparameter (allgemeine und spezielle postoperative Komplikationen, Gesamtmorbidität, Letalität, Qualität der TME (M.E.R.C.U.R.Y.-Kriterien), Anzahl der untersuchten Lymphknoten, onkologische Langzeitergebnisse) deskriptiv ausgewertet und hinsichtlich im Verlauf der Datenerfassung zu beobachtender Änderungen analysiert.

Die Datenauswertung erfolgte explorativ mit SPSS14. Zum Vergleich kategorialer Daten diente der $\mathrm{X}^{2}$-Test. Stetige Variablen wurden mit dem Student-t-Test oder bei mehr als 2 zu vergleichenden Gruppen mit der unifaktoriellen Varianzanalyse (one-

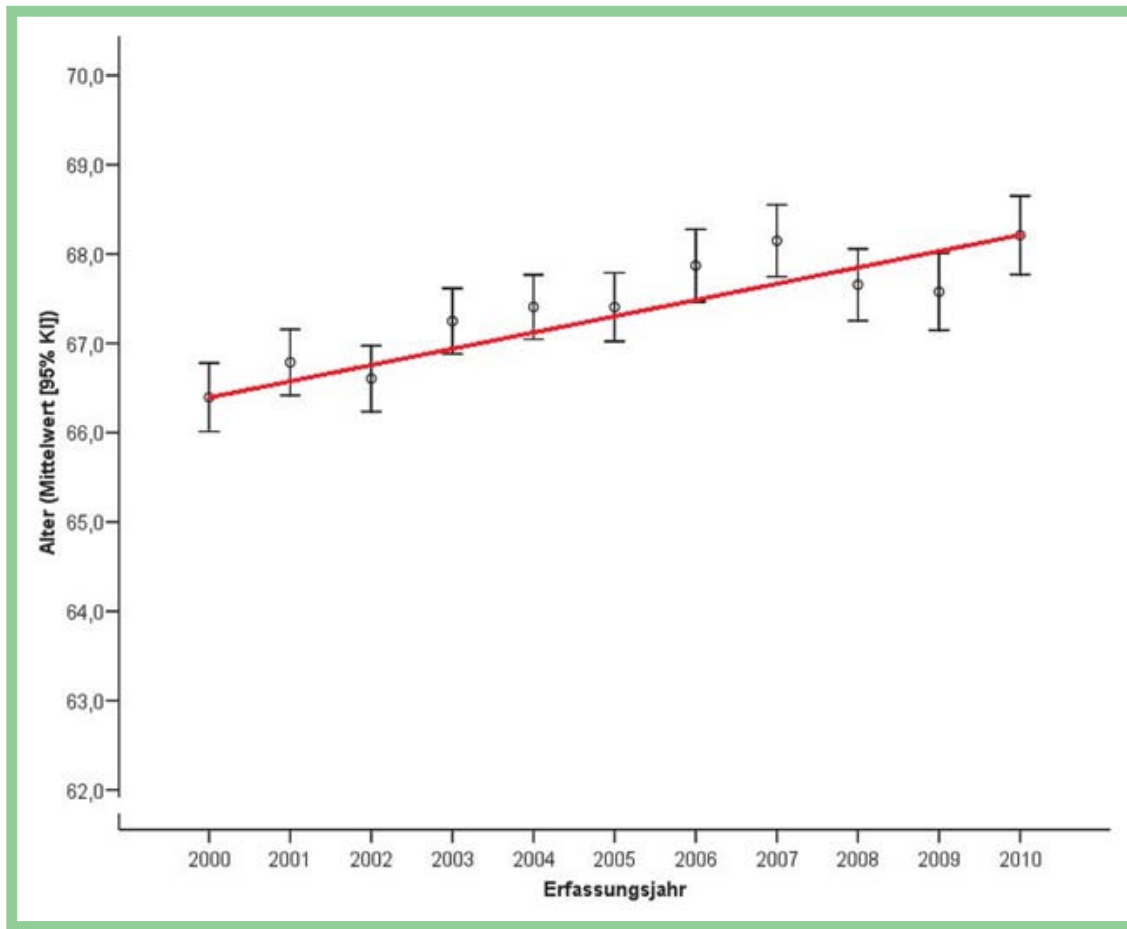

Abb. 1 Altersdurchschnitt der Patienten mit Tumorresektion (alle Resektionen) im Beobachtungsverlauf 2000-2010. 


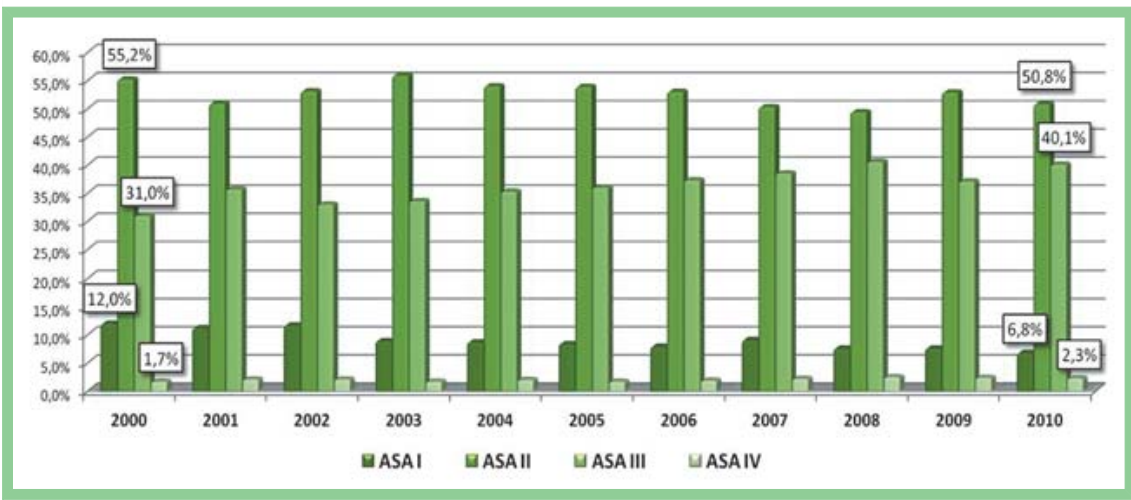

Abb. 2 Verteilung der ASA-Scores unter den Patienten mit Tumorresektion (alle Resektionen) im Studienverlauf 2000-2010.

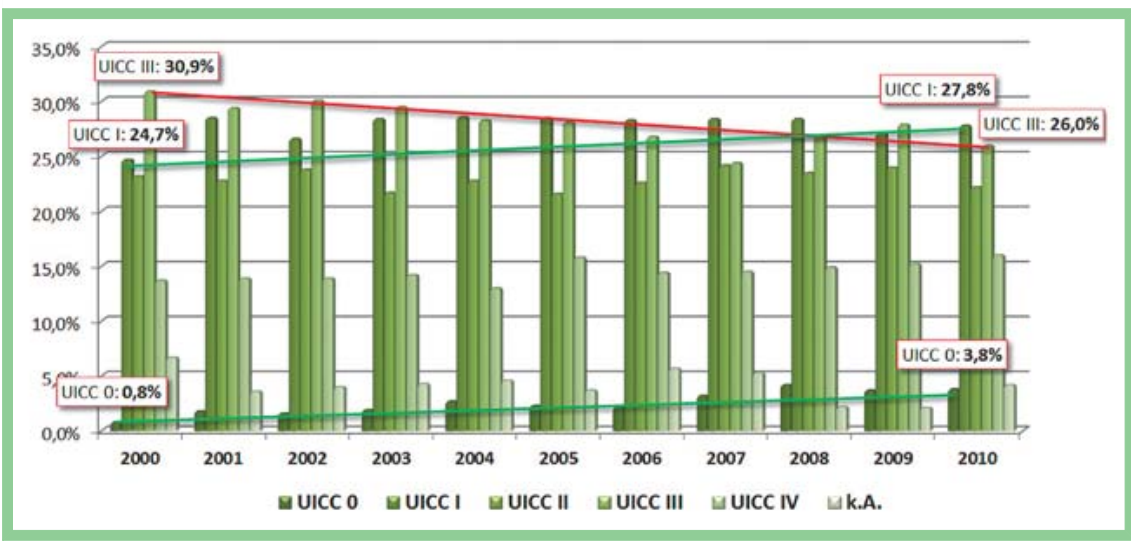

Abb. 3 Verteilung der postoperativen UICC-Stadien nach Tumorresektion (alle Resektionen) im Studienverlauf 2000-2010. Gemeinsame Darstellung der neoadjuvant behandelten und der nicht vorbehandelten Patienten.

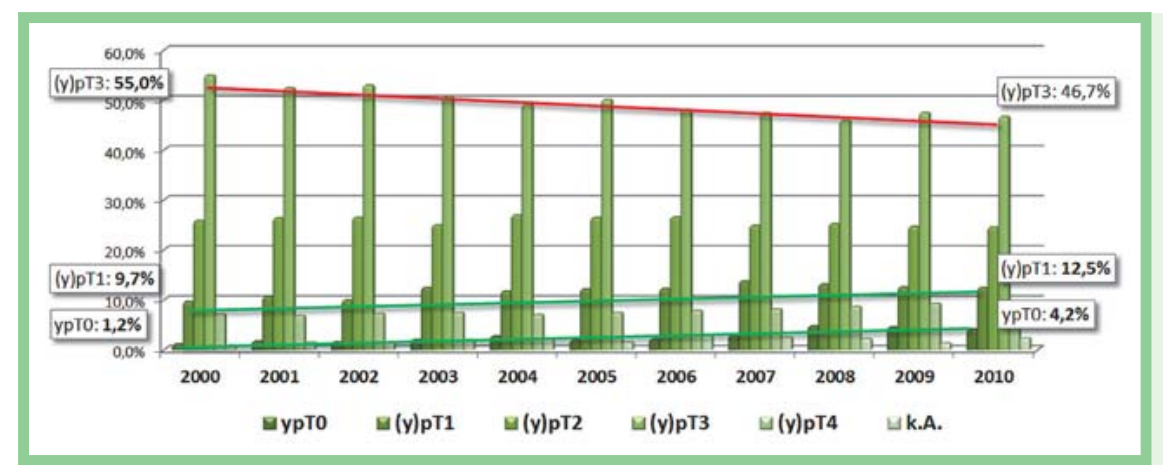

Abb. 4 Verteilung der pT-Kategorien nach Tumorresektion (alle Resektionen) im Studienverlauf 2000-2010. Gemeinsame Darstellung der neoadjuvant behandelten und der nicht vorbehandelten Patienten.

way ANOVA) verglichen und als Mittelwert und 95\%-Konfidenzintervall angegeben. Unterschiede zwischen den verglichenen Patientengruppen wurden bei einer Irrtumswahrscheinlichkeit $p<0,05$ als signifikant ausgewiesen. Für die Überlebensanalysen wurden Patienten, die im frühpostoperativen Verlauf verstorben waren, ausgeschlossen. Die univariate Überlebensanalyse erfolgte für die Lokalrezidivrate und das tumorfreie Überleben mit der Kaplan-Meier-Methode. Der Vergleich der Überlebensraten zwischen den Gruppen erfolgte mit dem Log-Rank-Test.

\section{Ergebnisse}

$\nabla$

Vom 01.01.2000 bis 31.12.2010 wurden 33724 Patienten mit einem Rektumkarzinom in der Qualitätssicherungsstudie „Rektumkarzinom“ erfasst. Bei 95,2\% Patienten erfolgte eine Resektion des Rektumkarzinoms. Bei 84,2\% dieser Patienten war die
Resektion des Karzinoms kurativ. Was die Tumorresektionsrate und die Rate kurativer Resektionen anbetrifft, so sind im Verlauf der Datenerfassung über die Jahre keine relevanten Schwankungen der Resektionsraten (93,9-96,8\% resp. 82,9-85,9\%) zu verzeichnen.

Hinsichtlich der Patientendemografie ist im Studienverlauf ein signifikanter Anstieg des Durchschnittalters der Patienten zu verzeichnen, bei denen eine Tumorresektion erfolgte ( $\triangle$ Abb. 1). Der Altersdurchschnitt der resezierten Patienten lag im Jahr 2000 bei 66,4 Jahren (95\%-KI: 66,0-66,8) und stieg auf 68,2 Jahre (95\%-KI: 67,8-68,6) im Jahr 2010 an ( $p<0,001)$. Gleichzeitig ist zu verzeichnen, dass im Verlauf ein zunehmender Anteil der resezierten Patienten in den höheren ASA-Scores klassifiziert wurde als es zu Beginn der Datenerfassung der Fall war. So lag der Anteil der ASA-III- und -VI-Patienten im Jahr 2000 bei 32,7\% und stieg im Beobachtungsverlauf auf 42,4\% im Jahr 2010 an (๑ Abb. 2; $\mathrm{p}<0,001)$. 


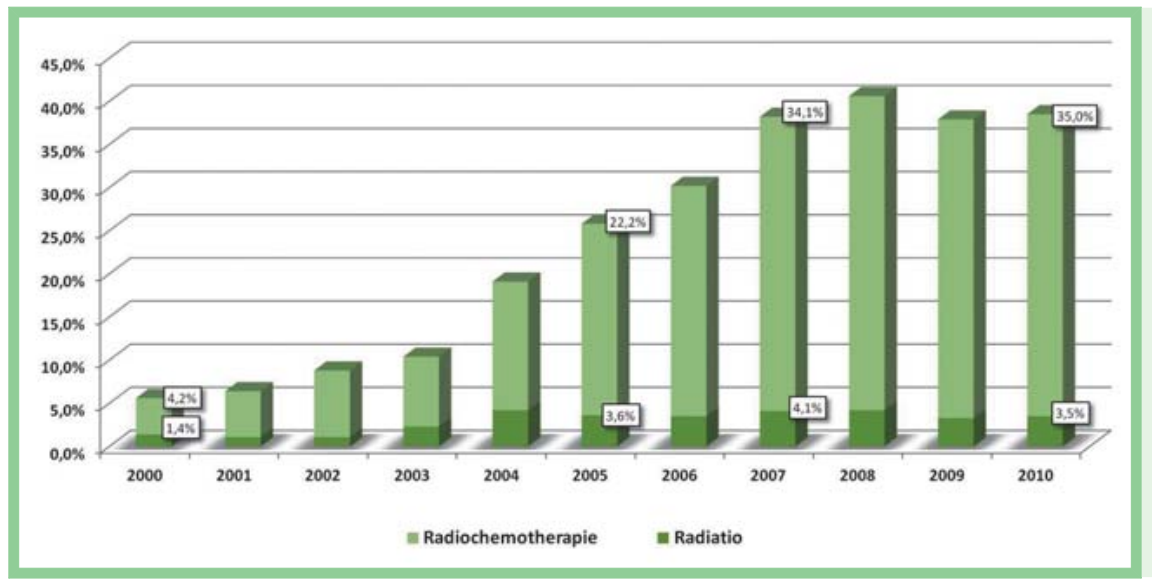

Abb. 5 Anteil der neoadjuvanten Bestrahlungen und Radiochemotherapien bei Patienten vor kurativer Tumorresektion (UICC-Stadien I-III) im Studienverlauf 2000-2010.

Hinsichtlich der Verteilung der Tumorstadien basierend auf dem postoperativen Staging ist bei gemeinsamer Darstellung der neoadjuvant behandelten und der nicht vorbehandelten Patienten ( Abb. 3) innerhalb der UICC-Stadien I-III im Verlauf ein geringer Shift zu den niedrigeren Tumorstadien zu verzeichnen. So nimmt der Anteil der UICC-III- sowie der UICC-II-Patienten von 31,0 resp. 23,1\% im Jahr 2000 auf 26,0 resp. 22,2\% im Jahr 2010 ab. Gleichzeitig steigt der Anteil der Patienten, bei denen nach neoadjuvanter Behandlung im Resektat histologisch kein Tumor mehr nachweisbar war („UICC 0“: 2000: 0,8\%; 2010: 3,8\%). Der Anteil der UICC-I-Patienten bleibt im Verlauf nahezu konstant. Die Verschiebungen innerhalb der UICC-Stadien I-III unter Einschluss der Patienten, bei denen in der postoperativen Histologie kein Tumor mehr nachweisbar war („UICC 0“), sind statistisch signifikant $(\mathrm{p}<0,001)$.

Bezüglich der Patienten, die bei Primärdiagnose des Rektumkarzinoms bereits eine synchrone Fernmetastasierung aufwiesen, ist im Verlauf der Datenerfassung eher eine geringe Zunahme der Häufigkeit zu verzeichnen. So stieg der Anteil der UICC-IV-Patienten von 13,7\% im Jahr 2000 auf 16,0\% im Jahr 2012 (๑ Abb. 3). Dieser Anstieg ist jedoch statistisch nicht signifikant $(p=0,079)$. Gleiche Veränderungen zeigen sich bei Betrachtung der pT-Kategorie wiederum bei zusammenfassender Darstellung der neoadjuvant behandelten und der nicht vorbehandelten Patienten ( Abb. 4). Während im Jahr 2000 noch 55,0\% der Karzinome als (y)pT3-Karzinome klassifiziert wurden, lag dieser Anteil 2010 nur noch bei 46,6\%. Bei einem im Vergleich unveränderten Anteil an (y)pT2-Karzinomen (2000: 25,9\%; 2010: 24,6\%) nahm der Anteil der ypT0- und (y)pT1-Karzinome zu (10,9\% vs. 16,7\%). Diese Verschiebungen sind statistisch signifikant $(\mathrm{p}<0,001)$.

Andererseits zeigt sich, dass der Anteil der (y)pT4-Karzinome im Verlauf gering, jedoch statistisch signifikant $(p=0,001)$ von $7,2 \%$ im Jahr 2000 auf 9,6\% im Jahr 2010 anstieg. Der Anteil der Karzinomresektate ohne Angabe der pT-Kategorie lag 2000 bei 0,9\%, im Jahr 2010 bei 2,6\%.

Hinsichtlich der multimodalen Therapie des Rektumkarzinoms ist, wie in $\triangle$ Abb. 5 dargestellt, festzustellen, dass der Anteil der neoadjuvant behandelten Karzinome - über alle Tumorhöhen und für nicht fernmetastasierte, resezierte Karzinome betrachtet - im Verlauf der Datenerfassung stetig von 5,6\% im Jahr 2000 auf 37,9\% im Jahr 2007 zunahm ( $\mathrm{p}<0,001$ ). Ab 2007 ist im weiteren Verlauf keine signifikante Änderung des Anteils der neoadjuvant behandelten Karzinome mehr festzustellen ( $p=0,077$ ).

Erst ab dem Jahr 2005 wurde die prätherapeutische T-Kategorie (cT) erfasst. In der $\bullet$ Abb. 6 ist der Anteil der neoadjuvant behan-

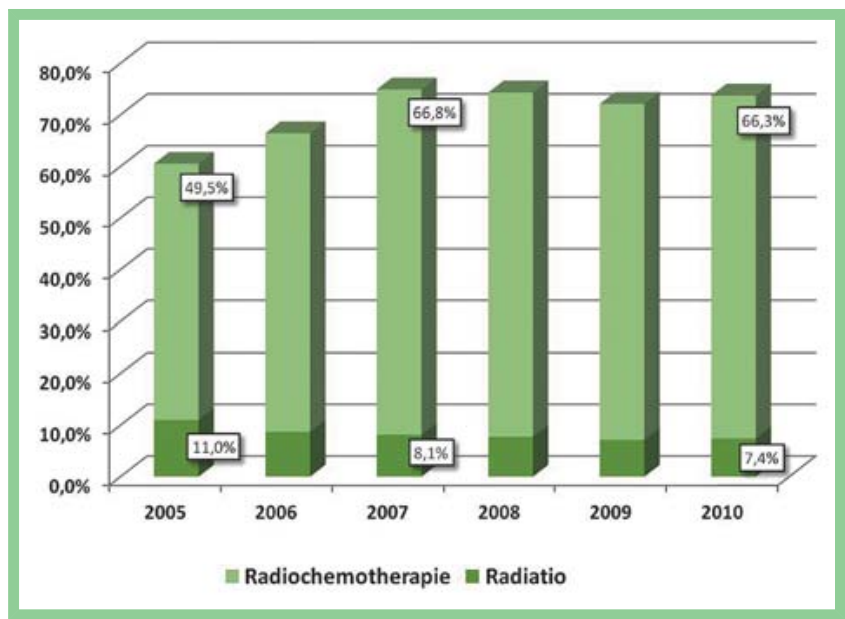

Abb. 6 Anteil der neoadjuvanten Bestrahlungen und Radiochemotherapien bei Patienten mit einem cT3-Rektumkarzinom vor kurativer Tumorresektion (keine Fernmetastasen) im Studienverlauf 2000-2010.

delten cT3-Rektumkarzinome (nicht fernmetastasierte, resezierte Karzinome der unteren zwei Rektumdrittel) dargestellt. Auch hier zeigt sich ein bis zum Jahr 2007 stetig steigender Anteil neoadjuvant behandelter cT3-Karzinome (2005: 60,5\%; 2007: 74,9\%; $\mathrm{p}<0,001)$. Ab dem Jahr 2007 ist der Anteil der neoadjuvant behandelten cT3-Karzinome konstant (2008: 74,3\%; 2009: 72,1\%; 2010: 73,7\%; $\mathrm{p}=0,605$ ).

Zur Bewertung der Effektivität einer neoadjuvanten Therapie werden in Tab. 1 für die Jahre 2009/2010 Patienten mit einem prätherapeutisch als cT3-Tumor klassifizierten, kurativ radikal resezierten Rektumkarzinom in den unteren zwei Dritteln in Abhängigkeit von der Art der neoadjuvanten Behandlung verglichen (UICC I-III). Wie die durchschnittlichen Tumordurchmesser zeigen, ist sowohl mit der alleinigen Bestrahlung als auch mit der Radiochemotherapie eine signifikante Tumorverkleinerung (down-sizing) im Vergleich zu den Patienten ohne neoadjuvante Therapie zu erreichen $(p<0,001)$. Dabei ist die neoadjuvante Radiochemotherpie signifikant effektiver als die alleinige Bestrahlung $(p<0,001)$. Die erreichte Tumorverkleinerung korreliert mit einem Shift hin zu niedrigeren T-Kategorien der Karzinome in der postoperativen histologischen Beurteilung nach neoadjuvanter Behandlung im Vergleich zu den nicht vorbehandelten Patienten. Dabei ist der Effekt nach Radiochemotherapie ebenfalls deutlicher als nach alleiniger Bestrahlung. 


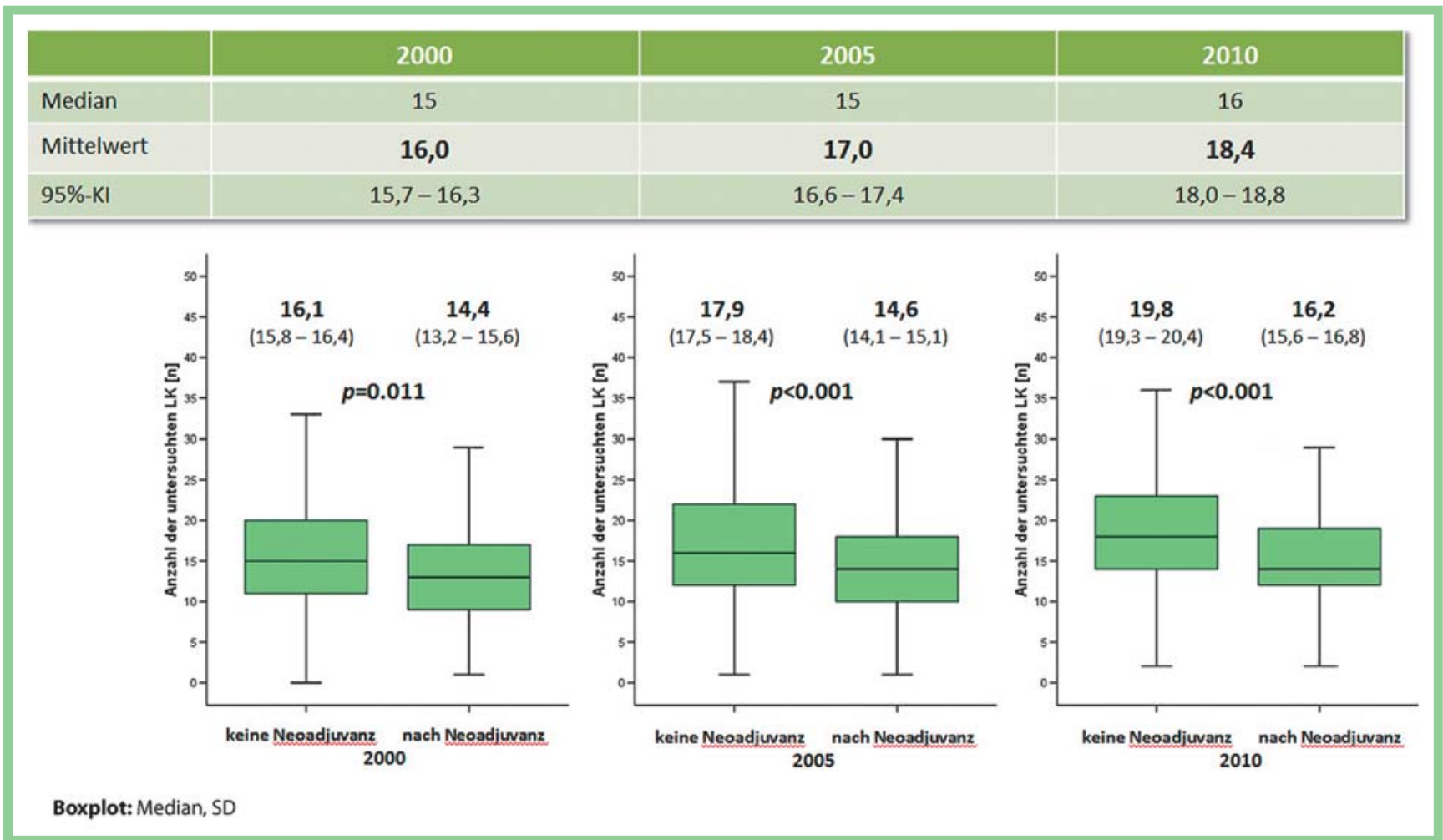

Abb. 7 Anzahl der untersuchten Lymphknoten nach Rektumresektion (gesamt, ohne Neoadjuvanz, nach neoadjuvanter Behandlung) im Studienverlauf $2000-2010$.

Tab. 1 Postoperative (y)pT-Kategorie und Tumordurchmesser [mm] der cT3-Karzinome (untere 2 Rektumdrittel) nach radikaler kurativer Resektion (UICC I bis III) in Abhängigkeit von der neoadjuvanten Behandlung; Jahrgänge 2009/2010.

\begin{tabular}{|c|c|c|c|c|c|}
\hline & $\begin{array}{l}\text { ohne Neo- } \\
\text { adjuvanz }\end{array}$ & $\begin{array}{l}\text { neoadjuvante } \\
\text { RTx }\end{array}$ & $\begin{array}{l}\text { neoadjuvante } \\
\text { RCTx (5-FU) }\end{array}$ & $\begin{array}{l}\text { neoadjuvante RCTx } \\
\text { (Capecitabine) }\end{array}$ & $\begin{array}{l}\text { neoadjuvante RCTx } \\
\text { (andere Chemo) }\end{array}$ \\
\hline \multicolumn{6}{|c|}{ (y)pT-Kategorie [\%] } \\
\hline (y)pт0 & - & $4,1 \%$ & $11,0 \%$ & $13,7 \%$ & $9,8 \%$ \\
\hline (y)pT1 & $1,5 \%$ & $2,1 \%$ & $6,9 \%$ & $9,8 \%$ & $7,8 \%$ \\
\hline (y)pT2 & $14,3 \%$ & $23,7 \%$ & $29,7 \%$ & $37,3 \%$ & $21,6 \%$ \\
\hline (y)pT3 & $78,1 \%$ & $64,9 \%$ & $50,1 \%$ & $37,3 \%$ & $60,8 \%$ \\
\hline (y)pT4 & $6,1 \%$ & $5,2 \%$ & $2,3 \%$ & $2,0 \%$ & - \\
\hline \multicolumn{6}{|c|}{ Tumordurchmesser [mm] } \\
\hline Mittelwert & 48,8 & 38,7 & 25,9 & 25,5 & 24,0 \\
\hline$(95 \%-K I)$ & $(46,7-50,8)$ & $(35,8-41,6)$ & $(24,7-27,0)$ & $(18,4-32,6)$ & $(20,7-27,4)$ \\
\hline
\end{tabular}

Hinsichtlich der Anzahl der vom Pathologen untersuchten Lymphknoten zeigt sich im Verlauf der Beobachtung ein signifikanter Anstieg $(\mathrm{p}<0,001)$. In der $\odot$ Abb. 7 sind die Jahrgänge 2000, 2005 und 2010 sowohl im Hinblick auf die insgesamt untersuchten Lymphknoten als auch bezogen auf die Anzahl der untersuchten Lymphknoten nach primärer Operation und nach neoadjuvanter Behandlung mit anschließender Resektion untersuchten Lymphknoten gegenübergestellt. Es zeigt sich im Verlauf ein signifikanter Anstieg der untersuchten Lymphknoten sowohl insgesamt $(p<0,001)$ als auch nach primärer Operation ( $p<0,001)$ und nach neoadjuvanter Behandlung mit anschließender Resektion ( $p<0,001)$. Es war weiterhin ein statistisch signifikanter Unterschied hinsichtlich der Anzahl der untersuchten Lymphknoten nach primärer Operation und nach neoadjuvanter Therapie in den untersuchten Jahrgängen nachzuweisen, wobei nach neoadjuvanter Behandlung im Durchschnitt 1,7 (Jahr 2000) bis 3,6 (Jahr 2010) Lymphknoten weniger untersucht wurden.
Tab. 2 TME-Qualität (Beurteilung durch den Pathologen nach den M.E.R.C.U.R.Y.-Kriterien) nach kurativer Resektion von Karzinomen in den unteren zwei Rektumdritteln (UICC I-III) der Jahre 2005-2010.

\begin{tabular}{lrrrrrr} 
TME-Qualität & $\mathbf{2 0 0 5}$ & $\mathbf{2 0 0 6}$ & $\mathbf{2 0 0 7}$ & $\mathbf{2 0 0 8}$ & $\mathbf{2 0 0 9}$ & $\mathbf{2 0 1 0}$ \\
Grad I & $81,7 \%$ & $82,7 \%$ & $82,0 \%$ & $82,5 \%$ & $85,3 \%$ & $86,9 \%$ \\
\hline Grad II & $15,9 \%$ & $13,2 \%$ & $14,1 \%$ & $15,0 \%$ & $12,4 \%$ & $10,1 \%$ \\
\hline Grad III & $2,4 \%$ & $4,1 \%$ & $3,9 \%$ & $2,5 \%$ & $2,3 \%$ & $3,0 \%$ \\
\hline
\end{tabular}

Die totale mesorektale Exzision als onkochirurgisches Grundprinzip bei der Resektion von in den unteren zwei Rektumdritteln lokalisierten Karzinomen kann in der flächendeckenden Versorgung heute als etabliert angesehen werden. Während zu Beginn der Datenerfassung im Jahr 2000 die TME-Rate noch bei 75,2\% lag, wurden im Jahr 2010 95,3\% der Rektumkarzinome in den unteren zwei Dritteln nach dem Prinzip der TME reseziert. 


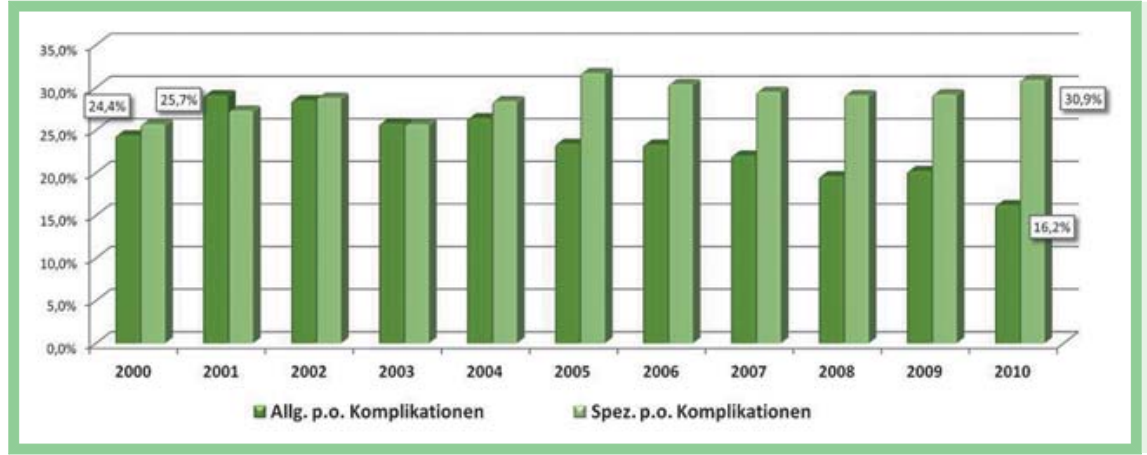

Abb. 8 Allgemeine und spezielle postoperative Komplikationen nach Rektumresektion im Studienverlauf $2000-2010$.
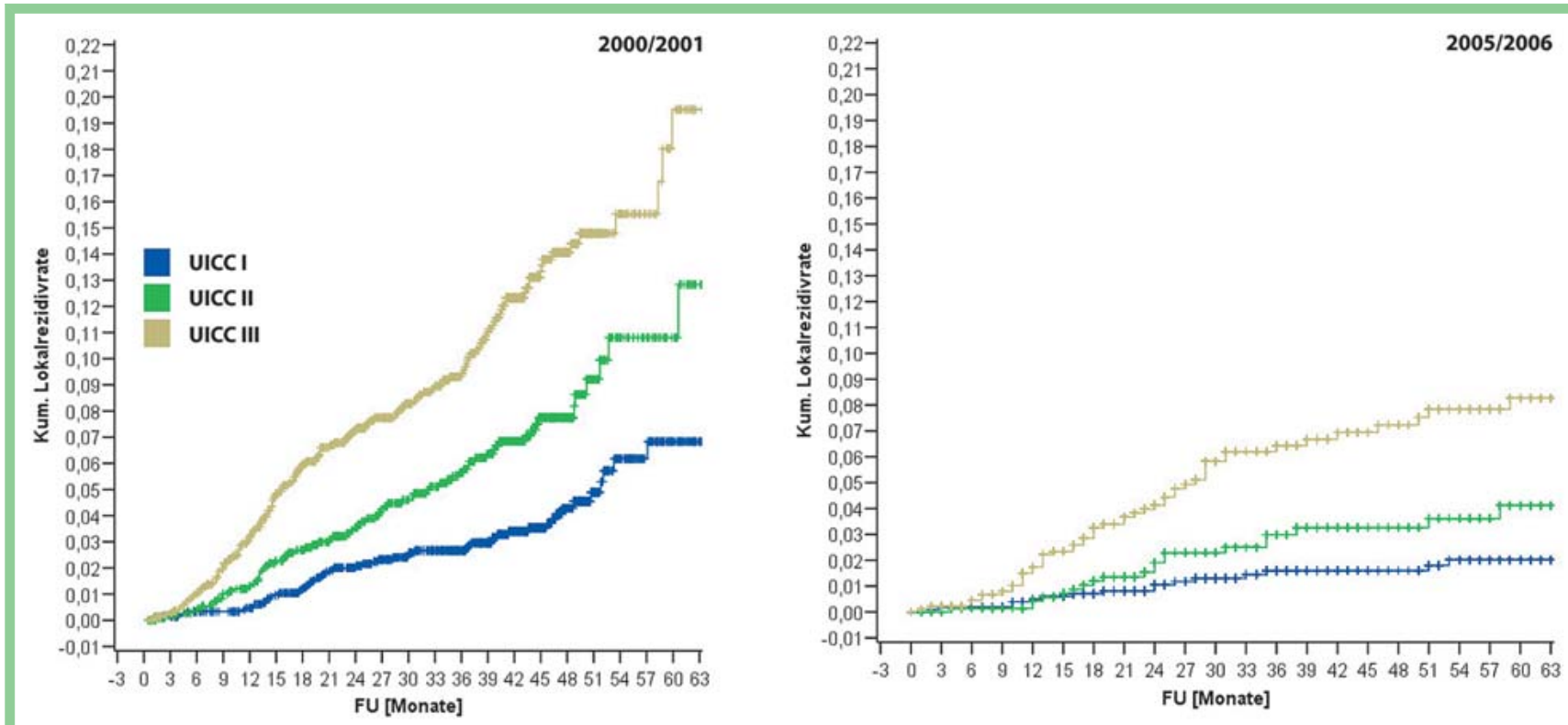

Abb. 9 5-Jahres-Lokalrezidivrate nach kurativer Resektion (UICC I-III) im Vergleich der Jahrgänge 2000/2001 vs. 2005/2006.

Tab. 3 Postoperative Gesamtmorbidität und Letalität im Studienverlauf 2000-2010.

\begin{tabular}{|c|c|c|c|c|c|c|c|c|c|c|c|}
\hline & 2000 & 2001 & 2002 & 2003 & 2004 & 2005 & 2006 & 2007 & 2008 & 2009 & 2010 \\
\hline Morbidität & $39,1 \%$ & $44,4 \%$ & $44,3 \%$ & $40,7 \%$ & $42,1 \%$ & $43,9 \%$ & $42,8 \%$ & $41,3 \%$ & $38,8 \%$ & $39,5 \%$ & $38,5 \%$ \\
\hline Letalität & $2,6 \%$ & $2,3 \%$ & $3,0 \%$ & $3,0 \%$ & $3,1 \%$ & $3,1 \%$ & $3,1 \%$ & $2,6 \%$ & $3,4 \%$ & $3,2 \%$ & $2,9 \%$ \\
\hline
\end{tabular}

Die Qualität der TME, die erst ab dem Jahr 2005 erfasst wurde, ist bei einem Anteil von 86,9\% Grad-I-TME und nur 3,0\% Grad-IIITME im Jahr 2010 als sehr gut einzustufen (@ Tab. 2).

Die Betrachtung der frühpostoperativen Ergebnisse in $\bullet \mathbf{A b b} . \mathbf{8}$ und Tab. 3 zeigt eine im Studienverlauf nahezu konstante postoperative Letalität nach Rektumkarzinomresektion mit einem Range zwischen 2,3\% und 3,4\%. Im Durchschnitt lag die postoperative Letalität bei $2,9 \%$. Auch für die postoperative Gesamtmorbidität ist festzustellen, dass diese über den gesamten Beobachtungsverlauf zwischen $38,5 \%$ und $44,4 \%$ nur gering variiert. Im Durchschnitt lag die postoperative Morbidität bei 41,6\%. Differenziert man die postoperative Morbidität nach allgemeinen und spezifischen postoperativen Komplikationen, so ist jedoch festzustellen, dass die allgemeinen postoperativen Komplikationen im Verlauf von 24,4\% im Jahr 2000 auf 16,2\% im Jahr 2010 signifikant gesunken sind (Durchschnitt: 23,8\%), wohingegen die spezifischen Komplikationen von 25,7\% im Jahr 2000 auf 30,9\% gestiegen sind (Durchschnitt: 28,7\%).

\section{Onkologische Langzeitergebnisse \\ $\nabla$}

Die onkologischen Langzeitergebnisse (Lokalrezidivrate, tumorfreies Überleben, Gesamtüberleben) wurden für die Behandlungszeiträume 2000/2001 vs. 2005/2006 verglichen. Die FURate der Patienten mit Einverständniserklärung zur FU-Datenerhebung betrug jeweils 80,1\% (2000/2001) und 85,5\% (2005/ 2006).

Nach kurativer Rektumkarzinomresektion in den UICC-Stadien IIII lag die 5-Jahres-Lokalrezidivrate der 2000/2001 resezierten Patienten insgesamt bei 11,7\%. Die 5-Jahres-Lokalrezidivrate der 2005/5006 resezierten Patienten war mit 4,6\% signifikant nied- 


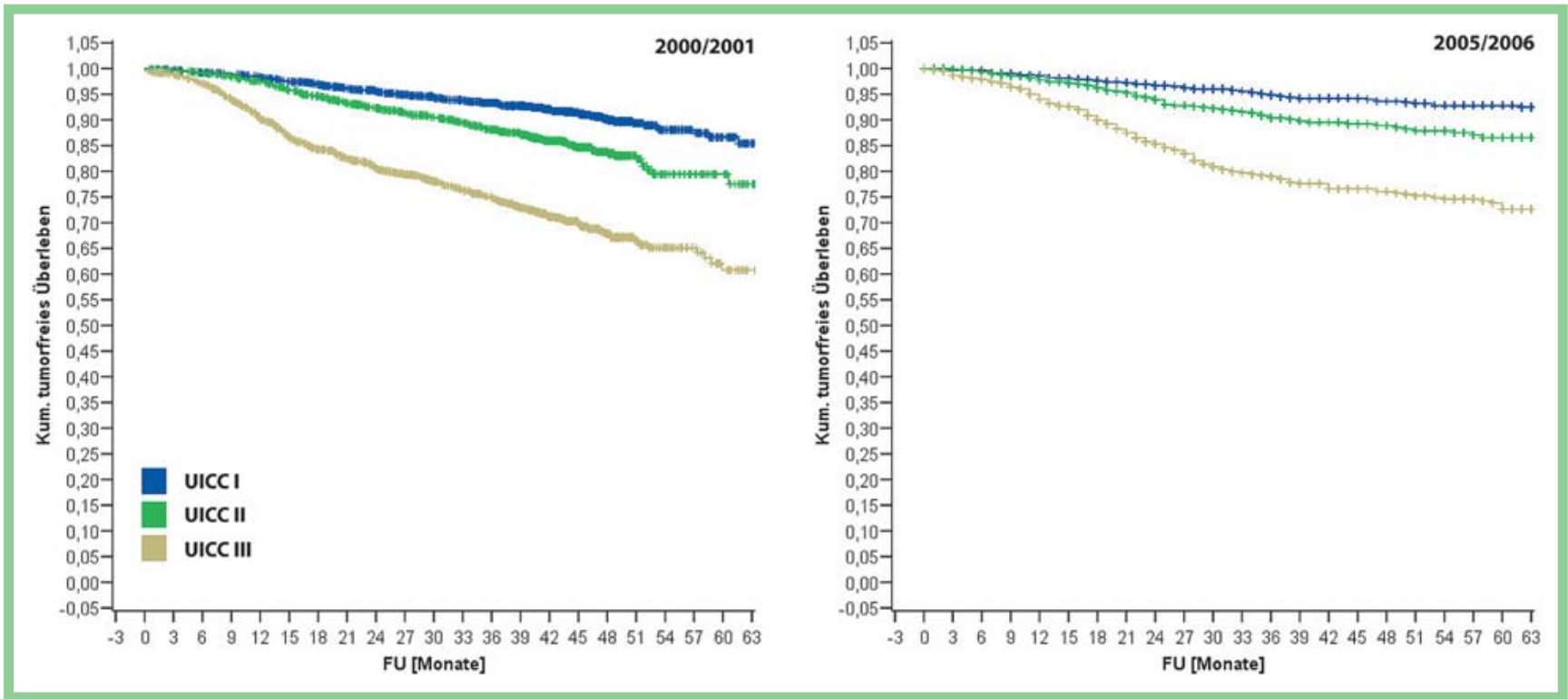

Abb. 10 Tumorfreies 5-Jahres-Überleben Rektumkarzinomresektion (UICC I-III) im Vergleich der Jahrgänge 2000/2001 vs. 2005/2006.
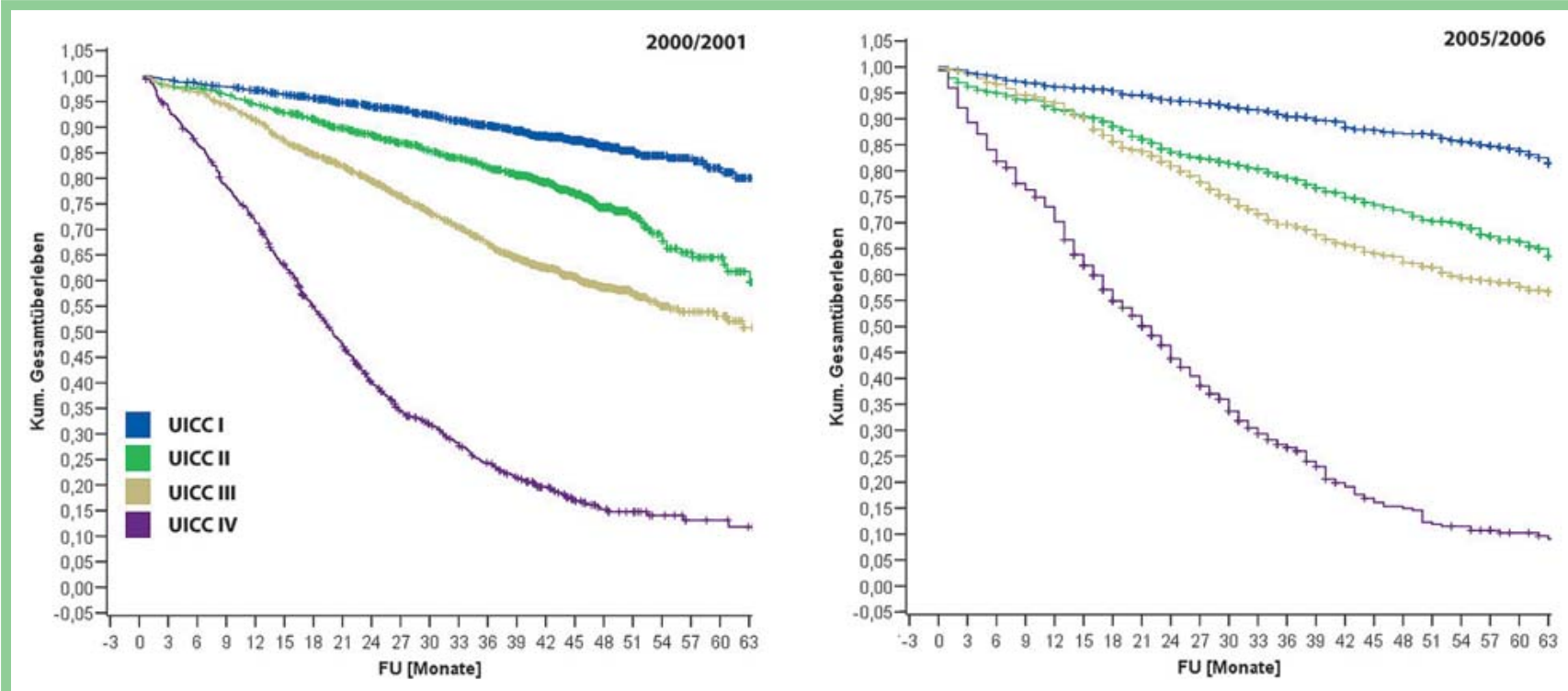

Abb. 11 5-Jahres-Gesamt-Überlebensrate nach Rektumkarzinomresektion (UICC I-IV) im Vergleich der Jahrgänge 2000/2001 vs. 2005/2006.

riger ( $\mathrm{p}<0,001)$. Die tumorfreie 5-Jahres-Überlebensrate betrug für die 2000/2001 kurativ resezierten Patienten 75,6\%. Die 2005/2006 resezierten Patienten wiesen mit 84,6\% eine signifikant höhere tumorfreie 5-Jahres-Überlebensrate auf $(\mathrm{p}<0,001)$. Das 5-Jahres-Overall-Survival aller operierten Patienten (UICC IIV) lag für die 2000/2001 operierten Patienten bei 58,1\% und für die 2005/2006 operierten Patienten bei 60,6\%. Hier ergab sich im Vergleich beider Zeiträume kein signifikanter Unterschied $(\mathrm{p}=0,776)$.

Der stadienbezogene Vergleich der 5-Jahres-Lokalrezidivraten nach kurativer Rektumkarzinomresektion in den UICC-Stadien I-III ( Abb. 9) zeigt, dass diese im Beobachtungszeitraum 2005/ 2006 mit 2,0\% (UICC I), 4,1\% (UICC II) und 8,3\% (UICC III) im Vergleich zu den Ergebnisse der Jahre 2000/2001 (UICC I: 6,8\%; UICC II: 10,8\%; UICC III: 18,3\%) für jedes UICC-Stadium signifikant geringer war (UICC I: $p=0,001$; UICC II: $p<0,001$; UICC III: $\mathrm{p}<0,001)$. Ebenso zeigt der stadienbezogene Vergleich der tumorfreien 5-Jahres-Überlebensraten nach kurativer Resektion ( $\triangle$ Abb. 10) ein für jedes Stadium günstigeres Outcome der 2005/2006 resezierten Patienten (UICC I: 92,8\%; UICC II: 86,6\%; UICC III: 72,6\%) im Vergleich zu den 2000/2001 resezierten Patienten (UICC I: 86,6\%; p =0,004; UICC II: 77,5\%; p =0,006; UICC III: $60,8 \%$; $<0,001)$.

Der Vergleich der 5-Jahres-Gesamtüberlebensraten beider Beobachtungszeiträume innerhalb der jeweiligen UICC-Stadien ( Abb. 11) zeigt hingegen keine signifikanten Unterschiede (UICC I: $p=0,547$; UICC II: $p=0,250$; UICC III: $p=0,072$; UICC IV: $\mathrm{p}=0,592$; Abb. 11). 81,9 vs. 83,7\% der Patienten im UICC-Stadium I, 64,8 vs. 66,3\% der Patienten im UICC-Stadium II, 53,1 vs. $57,6 \%$ der Patienten im UICC-Stadium III und 11,2 vs. 9,2\% der Patienten im UICC-Stadium IV überlebten 5 Jahre. 


\section{Diskussion}

Ausgehend von ca. 25000 an einem Rektumkarzinom jährlich Neuerkrankten in Deutschland spiegeln die in der prospektiven multizentrischen Beobachtungsstudie erfassten und hier analysierten Patienten ca. ein Siebtel der jährlich in Deutschland operativ behandelten Rektumkarzinompatienten wider. Die im Rahmen der Studie erfassten Patienten rekrutieren sich aus Kliniken, die das gesamte Versorgungsprofil - vom Krankenhaus der Grund- und Regelversorgung bis hin zur Universitätsklinik - vertreten. Die Vollständigkeit der Erfassung der in den teilnehmenden Kliniken behandelten Patienten wurde durch einen regelmäßigen Abgleich der erfassten Patienten mit den KIS-Daten der jeweiligen Kliniken überprüft [17]. Allerdings bezieht sich der Abgleich nur auf operierte, nicht auf die ausschließlich palliativ, nicht operativ behandelten Patienten. Die erfassten Daten wurden bei Eingabe in die Datenbank sowie vor Abschluss eines Erfassungsjahrs auf Plausibilität geprüft. Nicht plausible Daten wurden in den jeweiligen Kliniken hinterfragt.

Mit dem Instrument der prospektiven multizentrischen Beobachtungsstudie sind Limitierungen verbunden, die bei der Wertung der Ergebnisse zu berücksichtigen sind [18]. Die hier vorgestellten Ergebnisse geben einen Einblick in die Versorgungsqualität von Rektumkarzinompatienten in Deutschland.

Zunächst zeigt die Betrachtung der patientenbezogenen Parameter Alter und ASA-Score, dass die im Verlauf der QS-Studie erfassten Patienten signifikant älter wurden und mit zunehmend mehr Komorbiditäten belastet sind. Dabei ist zu beachten, dass es sich bei den betrachteten Patienten ausschließlich um tumorresezierte Patienten handelt. Entsprechend ist diese Entwicklung dahingehend zu werten, dass die Indikation zur Tumorresektion zunehmend auch bei älteren, mit mehr Komorbiditäten belasteten Patienten gestellt wird. Trotz des signifikant gestiegenen Alters der resezierten Patienten und dem zunehmend höheren Anteil an ASA-III/IV-Patienten war die Rate an allgemeinen postoperativen Komplikationen im Verlauf der Datenerfassung signifikant rückläufig. Dies spricht für ein optimiertes, an das veränderte Patientengut angepasstes perioperatives Management. Im Gegensatz zu den allgemeinen postoperativen Komplikationen zeigen die spezifischen postoperativen Komplikationen keine rückläufige Tendenz. Die Rate an spezifischen postoperativen Komplikationen ist im Verlauf der analysierten Jahrgänge eher konstant bis gering ansteigend. In der Summe ist im Verlauf der Datenerfassung die postoperative Morbidität konstant geblieben. Gleichermaßen zeigt sich für die postoperative Letalität keine relevante Änderung im Verlauf der Datenerfassung.

In den letzten Jahrzehnten hat sich die Behandlung von Patienten mit einem Rektumkarzinom grundlegend gewandelt [19, 20]. Die Etablierung der veränderten Therapiekonzepte lässt sich anhand der Daten der QS-Studie auch in der flächendeckenden Versorgung nachweisen. So stieg der Anteil der bei kurativem Therapieansatz neoadjuvant behandelten Rektumkarzinome im Verlauf der Datenerfassung signifikant auf 38,5\% im Jahr 2010 an. Insbesondere bei Betrachtung der prätherapeutisch als cT3-Karzinome klassifizierten Tumoren der unteren zwei Rektumdrittel lag der Anteil der neoadjuvant behandelten Patienten bei 73,7\% im Jahr 2010. Diese Entwicklung zur multimodalen Therapie der fortgeschrittenen Karzinome zeigen auch Kohortenanalysen aus anderen Ländern [21].

Vor diesem Hintergrund sind die Veränderungen in der Verteilung der Tumorstadien zu betrachten.
Es zeigt sich, dass der Anteil der UICC-III-Patienten im Verlauf um $5 \%$ signifikant abnahm. Korrespondierend dazu findet sich eine stetige Zunahme des Anteils der Patienten, bei denen postoperativ, bei prätherapeutisch gesichertem Karzinom und nach erfolgter Neoadjuvanz kein Karzinom mehr nachweisbar war, bei nahezu gleich gebliebenem Anteil von UICC-I- und UICC-II-Patienten. Eine nahezu identische Entwicklung zeigt sich bei Betrachtung der T-Kategorien. Bei um 8,4\% abnehmendem Anteil an pT3-Patienten und um 1,3\% abnehmendem Anteil von pT2-Patienten stieg der Anteil der pT1-Patienten um 2,8\% sowie der Anteil der Patienten, bei denen postoperativ kein Tumor mehr nachzuweisen war, um 3\%. Bei der Wertung dieser Entwicklungen ist jedoch zu berücksichtigen, dass es sich bei den UICC-Stadien und den TKategorien um postoperative Tumorklassifizierungen handelt. Es darf daher nicht uneingeschränkt auf einen Effekt der sich zunehmend etablierten neoadjuvanten Behandlung rückgeschlossen werden. Hierin liegt eine wesentliche Limitierung der Studie, die im Verlauf der Datenerfassung durchgehend nur die postoperative Tumorklassifikation erfasste, was die Aussagefähigkeit einschränkt. Erst seit 2005 werden die präoperativen cT-Kategorien mit erfasst. Allerdings kann unter Berücksichtigung des Anteils der Patienten, die im UICC-Stadium IV erstdiagnostiziert wurden und deren Anteil im Verlauf der Datenerfassung keine signifikante Änderung - eher eine tendenzielle Zunahme zeigt, auf einen Einfluss des häufigeren Einsatzes neoadjuvanter Therapien hinsichtlich der Verschiebungen in den UICC-Stadien I bis III rückgeschlossen werden.

Bei der Analyse der Patienten mit einem klinisch als cT3-Karzinom klassifizierten Rektumkarzinom zeigt der Vergleich der neoadjuvant behandelten Patienten und der primär operierten Patienten, dass Patienten nach neoadjuvanter Radiochemotherapie in der definitiven histopathologischen Beurteilung signifikant kleinere Tumordurchmesser haben und eine signifikante Verschiebung hin zu den geringeren T-Kategorien resultiert. Bei 9,8-13,7\% der Patienten wurde durch die neoadjuvante Radiochemotherapie sogar eine komplette Tumorremission erreicht. Die jüngst von Rödel et al. [22] publizierten Ergebnisse einer multizentrischen, randomisierten Phase-III-Studie zeigen für die 5FU-basierte neoadjuvante Radiochemotherapie eine vergleichbare Rate vollständiger Remissionen von 13\%. Bei Intensivierung der neoadjuvanten Radiochemotherapie mit Oxaliplatin konnte die Rate an kompletten Remissionen sogar signifikant auf $17 \%$ gesteigert werden.

In verschiedenen Analysen wurde gezeigt, dass nach neoadjuvanter Radiatio die Anzahl der vom Pathologen beurteilten Lymphknoten signifikant sinkt [23-25]. Auch in der vorgestellten Auswertung ist die Anzahl der vom Pathologen untersuchten Lymphknoten nach neoadjuvanter Bestrahlung in den untersuchten Jahrgängen 2000, 2005 und 2010 jeweils signifikant geringer als die Anzahl der untersuchten Lymphknoten bei den Patienten, die nicht neoadjuvant behandelt wurden. Trotz des zunehmenden Anteils neoadjuvant behandelter Patienten zeigt sich jedoch im Verlauf eine signifikante Steigerung der Anzahl untersuchter Lymphknoten sowohl bei den neoadjuvant behandelten als auch den nicht neoadjuvant behandelten Patienten. Im Ergebnis stieg im Verlauf die durchschnittliche Anzahl der untersuchten Lymphknoten bei Betrachtung aller Patienten von 16,0 im Jahr 2000 auf 18,4 im Jahr 2010, was für die zunehmende Qualität auch der pathologischen Diagnostik spricht.

Parallel zur Etablierung der neoadjuvanten Behandlung von Rektumkarzinomen hat sich die Qualität der Chirurgie verbessert. Seit 2005 wird nahezu jeder Patient mit einem tiefsitzenden Kar- 
zinom dem von Heald [1] inaugurierten Konzept der TME folgend operiert. Dabei ist die im Jahr 2010 erreichte Qualität der TME bei 86,9\% Grad-I-Resektionen und nur 3,0\% Grad-III-Resektionen im Vergleich zu den Ergebnissen anderer Studien als sehr hoch zu bewerten $[10,26]$.

Im Ergebnis der Etablierung und Optimierung der Therapiekonzepte wurde in der flächendeckenden Versorgung von Patienten mit Rektumkarzinomen eine signifikante Verbesserung der onkologischen Langzeitergebnisse im Hinblick auf die lokale Tumorkontrolle erreicht. So konnte die 5-Jahres-Lokalrezidivrate nach kurativer Resektion (UICC I-III) von 11,7\% (2000/2001) auf 4,6\% (2005/2006) und damit auf weniger als die Hälfte reduziert werden. Diese signifikante Senkung der Lokalrezidivrate lässt sich für jedes einzelne UICC-Stadium nachweisen und ist damit nicht nur allein auf die neoadjuvante Behandlung zurückzuführen, die sich erst ab 2005 etabliert hat, sondern in hohem Maße auf die Optimierung der chirurgischen Resektionstechnik. Dass die Optimierung der chirurgischen Technik einen entscheidenden Einfluss hat, wird nicht zuletzt durch die von Heald publizierten Ergebnisse [1], aber auch durch die Ergebnisse der Swedish-RectalCancer-Study [27] gestützt.

Gleichermaßen lässt sich für das tumorfreie Überleben nach kurativer Resektion eine signifikante Verbesserung des Outcome verzeichnen. Auch hier lässt sich für jedes UICC-Stadium eine signifikante Steigerung der tumorfreien 5-Jahres-Überlebensrate nachweisen.

Trotz verbesserter lokaler Tumorkontrolle und höherer tumorfreier Überlebensrate infolge der Therapieoptimierung beim Rektumkarzinom lässt sich keine Änderung des Langzeitüberlebens verzeichnen. Dies ist aus den prospektiven randomisierten Studien zur neoadjuvanten Therapie beim Rektumkarzinom bekannt $[3,28,29]$. Auch wenn in der Auswertung der 12-Jahres-Ergebnisse der holländischen TME-Studie ein positiver Effekt der Neoadjuvanz nicht nur auf die lokale Tumorkontrolle, sondern auch auf das Langzeitüberleben gezeigt werden konnte [9], ist ein verbessertes Gesamtüberleben für die den vorgestellten Daten zugrunde liegende flächendeckende Versorgung nicht nachweisbar.

\section{Interessenkonflikt: Nein}

\section{Literatur}

1 Heald RJ, Moran BJ, Ryall RD et al. Rectal cancer: the Basingstoke experience of total mesorectal excision, 1978-1997. Arch Surg 1998; 133 : 894-899

2 Sauer R, Becker H, Hohenberger W et al. Preoperative versus postoperative chemoradiotherapy for rectal cancer. N Engl J Med 2004; 351: $1731-1740$

3 Sauer R, Liersch T, Merkel S et al. Preoperative versus postoperative chemoradiotherapy for locally advanced rectal cancer: results of the German CAO/ARO/AIO-94 randomized phase III trial after a median follow-up of 11 years. J Clin Oncol 2012; 30: 1926-1933

4 Kapiteijn E, Marijnen CA, Nagtegaal ID et al. Preoperative radiotherapy combined with total mesorectal excision for resectable rectal cancer. N Engl J Med 2001; 345: 638-646

5 Folkesson J, Birgisson H, Pahlman L et al. Swedish Rectal Cancer Trial: long lasting benefits from radiotherapy on survival and local recurrence rate. J Clin Oncol 2005; 23: 5644-5650

6 Cedermark B, Johansson H, Rutqvist LE et al. The Stockholm I trial of preoperative short term radiotherapy in operable rectal carcinoma. A prospective randomized trial. Stockholm Colorectal Cancer Study Group. Cancer 1995; 75: 2269-2275

7 Hermanek P, Mansmann U, Altendorf-Hofmann et al. Vergleichende Beurteilung der onkologischen Ergebnisqualität beim colorectalen Carcinom. Klinikvergleiche anhand von Surrogatendpunkten? Chirurg 1999; 70: 407-414
8 Kapiteijn E, Marijnen CA, Colenbrander AC et al. Local recurrence in patients with rectal cancer diagnosed between 1988 and 1992: a population-based study in the west Netherlands. Eur J Surg Oncol 1998; 24 : 528-535

9 van Gijn W, Marijnen CA, Nagtegaal ID et al. Preoperative radiotherapy combined with total mesorectal excision for resectable rectal cancer: 12-year follow-up of the multicentre, randomised controlled TME trial. Lancet Oncol 2011; 12: 575-582

10 Nagtegaal ID, van de Velde CJ, van der Worp E et al. Macroscopic evaluation of rectal cancer resection specimen: clinical significance of the pathologist in quality control. J Clin Oncol 2002; 20: 1729-1734

11 Schmitz KJ, Chmelar C, Berg E et al. Pathological work-up of rectal cancer following partial/total mesorectal excision. Pathologe 2011; 32: 321-329

12 Nagtegaal ID, Quirke $P$. What is the role for the circumferential margin in the modern treatment of rectal cancer? J Clin Oncol 2008; 26: 303312

13 Garlipp B, Ptok H, Schmidt U et al. Neoadjuvant chemoradiotherapy for rectal carcinoma: effects on anastomotic leak rate and postoperative bladder dysfunction after non-emergency sphincter-preserving anterior rectal resection. Results of the Quality Assurance in Rectal Cancer Surgery multicenter observational trial. Langenbecks Arch Surg 2010; 395: 1031-1038

14 Ptok H, Marusch F, Meyer et al. Impact of anastomotic leakage on oncological outcome after rectal cancer resection. Br J Surg 2007; 94: 15481554

15 Bittner R, Burghardt J, Gross E et al. Qualitätsindikatoren bei der Diagnostik und Therapie des Rektumkarzinoms. Zentralbl Chir 2007; 132: 85-94

16 Gastinger I, Marusch F, Steinert R et al. Protective defunctioning stoma in low anterior resection for rectal carcinoma. Br J Surg 2005; 92: $1137-1142$

17 Marusch F, Koch A, Schmidt $U$ et al. Stellenwert der Rektumexstirpation im Therapiekonzept des tief sitzenden Rektumkarzinoms. Chirurg 2003; 74: 341-352

18 Concato J, Shah N, Horwitz RI. Randomized, controlled trials, observational studies, and the hierarchy of research designs. N Engl J Med 2000; 342: 1887-1892

19 Ceelen WP. Progress in rectal cancer treatment. ISRN Gastroenterol 2012; 2012: 64818

20 Gaedcke J, Liersch T, Hess C et al. [Rectal cancer: current status of multimodal therapy-when and how?]. Zentralbl Chir 2011; 136: 334-342

21 Renouf DJ, Woods R, Speers C et al. Improvements in 5-year Outcomes of Stage II/III Rectal Cancer Relative to Colon Cancer. Am J Clin Oncol 2012 Aug 2 [Epub ahead of print]

22 Rödel C, Liersch T, Becker $H$ et al. Preoperative chemoradiotherapy and postoperative chemotherapy with fluorouracil and oxaliplatin versus fluorouracil alone in locally advanced rectal cancer: initial results of the German CAO/ARO/AIO-04 randomised phase 3 trial. Lancet Oncol 2012; 13: 679-687

23 Le M, Nelson R, Lee W et al. Evaluation of lymphadenectomy in patients receiving neoadjuvant radiotherapy for rectal adenocarcinoma. Ann Surg Oncol 2012; 19: 3713-3718

24 Damin DC, Rosito MA, Contu PC et al. Lymph node retrieval after preoperative chemoradiotherapy for rectal cancer. J Gastrointest Surg 2012; 16: $1573-1580$

25 Awwad GE, Tou SI, Rieger NA. Prognostic significance of lymph node yield after long-course preoperative radiotherapy in patients with rectal cancer: a systematic review. Colorectal Dis 2013; 15: 394-403

26 Quirke P, Steele R, Monson J et al. Effect of the plane of surgery achieved on local recurrence in patients with operable rectal cancer: a prospective study using data from the MRC CR07 and NCIC-CTG CO16 randomised clinical trial. Lancet 2009; 373: 821-828

27 Martling AL, Holm T, Rutqvist LE et al. Effect of a surgical training programme on outcome of rectal cancer in the county of Stockholm. Lancet 2000: 356: 93-96

28 Peeters KC, Marijnen CA, Nagtegaal ID et al. The TME trial after a median follow-up of 6 years: increased local control but no survival benefit in irradiated patients with resectable rectal carcinoma. Ann Surg 2007; 246: 693-701

29 Sebag-Montefiore D, Stephens RJ, Steele et al. Preoperative radiotherapy versus selective postoperative chemoradiotherapy in patients with rectal cancer (MRC CR07 and NCIC-CTG C016): a multicentre, randomised trial. Lancet 2009; 373: 811-820 